\title{
Non-Tumoral Factors Affecting The Preference of Nephron-Sparing Surgery in The Treatment of Stage 1 Renal Cell Carcinoma Patients in Turkey
}

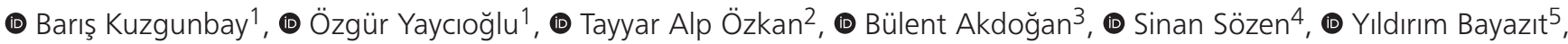

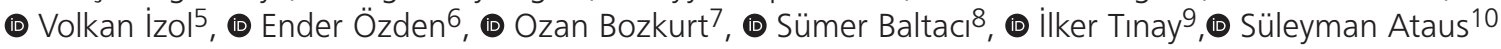 \\ ${ }^{1}$ Başkent University Faculty of Medicine, Department of Urology, Adana, Turkey \\ ${ }^{2}$ Acıbadem Hospital, Clinic of Urology, Kocaeli, Turkey \\ 3 Hacettepe University Faculty of Medicine, Department of Urology, Ankara, Turkey \\ ${ }^{4}$ Gazi University Faculty of Medicine, Department of Urology, Ankara, Turkey \\ ${ }^{5}$ Çukurova University Faculty of Medicine, Department of Urology, Adana, Turkey \\ ${ }^{6}$ Ondokuz Mayıs University Faculty of Medicine, Department of Urology, Samsun, Turkey \\ 7Dokuz Eylül University Faculty of Medicine, Department of Urology, Izmir, Turkey \\ ${ }^{8}$ Ankara University Faculty of Medicine, Department of Urology, Ankara, Turkey \\ ${ }^{9}$ Anadolu Medical Center, Clinic of Urology, Istanbul, Turkey \\ 10Istanbul Forte Urology Center, Clinic of Urology, Istanbul, Turkey \\ ${ }^{11 D r}$. Members of Kidney Cancer Study Group
}

\begin{abstract}
Objective: Nephron-sparing surgery (NSS) is the first-line treatment for T1N0M0 renal tumors (RT). The aim of this multicentric retrospective study is to investigate the national trends and the effect of non-tumoral factors in the preference of NSS as treatment of T1 RT in Turkey.

Materials and Methods: Relevant data for patients operated between 1997 and 2017 was collected from the Urologic Cancer Database-Kidney Urooncology Association, Turkey (UroCaD-K).

Results: We included 3195 T1NOMO RT patients in this study. There was a significant increase in the number of NSS performed with time, $9.26 \%$ between $1997-$ 2002 to $54.78 \%$ between $2013-2017$ ( $p<0.001$ ). NSS proportion decreased with increasing age $(p<0.001)$; but increased with better hospital facility ( $p<0.001$ ). From multivariate analysis; younger age, later operation date, larger hospital size with higher nephrectomy centers like university hospitals were independently associated non-tumoral factors favoring NSS over radical nephrectomy (RN).

Conclusion: We observed significant disparity in the use of NSS for T1 RT among the elderly ( $>61$ years), small hospital size ( $\leq 500$ beds), lower nephrectomy volume (<100 nephrectomies/year), and Non-University Hospitals. This disparity can be resolved by persistent education of the residents and urologists with periodic courses and practical training, increasing the funds and strengthening the technical equipment of centers, thereby favoring the performance of NSS even in smaller centers. This will ensure that suitable patients are treated with NSS rather than RN, regardless of the hospital type.
\end{abstract}

Keywords: Surgical treatment, renal cell carcinoma, nephron-sparing surgery, non-tumor factors, nephrectomy volume

\section{Introduction}

Renal cell carcinoma (RCC) represents $2-3 \%$ of all cancers. There is a 1.5:1 male predominance, with a peak incidence between 60 and 70 years of age. Smoking, obesity, hypertension, and having a first-degree relative with RCC are known etiological risk factors for RCC. Data from Europe and the United States show that the incidence of RCC increased by about $2 \%$ over the last two decades, probably due to an increased use of and advancement in radiologic imaging such as ultrasonography, computed tomography, and magnetic resonance imaging. This has also caused an increase in the proportion of incidentally diagnosed small low-stage tumors (1). 
The tumor node metastasis (TNM) classification is recommended for the staging of RCC (1). According to the 2017 TNM classification; T1 is defined as a tumor $<7 \mathrm{~cm}$ or less in the greatest dimension, limited to the kidney. Moreover, T1 is divided into two: T1a if tumor $<4 \mathrm{~cm}$ or less; T1b if tumor $>4 \mathrm{~cm}$ but $<7 \mathrm{~cm}$ (1). The European Association of Urology Guidelines strongly recommend nephron-sparing surgery (NSS) as the first-line treatment of T1 renal tumors (RT) since radical nephrectomy (RN) and NSS have similar oncological outcomes as well as safety and complication rates independent of the surgical technique; be it open, laparoscopic or robotic surgery $(1,2,3,4)$. However, many systematic reviews and metaanalyses showed overall survival, cardiac-specific survival, renal reserve, and quality of life benefits in favor of NSS $(4,5,6,7,8,9)$. A recently published study concluded that the prognostic risk of chronic kidney disease in patients with kidney cancer increases when the preoperative glomerular filtration rate is less than $60 \mathrm{~mL} / \mathrm{min} / 1.73 \mathrm{~m}^{2}$ or the postoperative rate is less than 45 $\mathrm{mL} / \mathrm{min} / 1.73 \mathrm{~m}^{2}(10)$. It was also emphasized that additional factors, including non-surgical causes of chronic kidney disease and the degree of albuminuria, can also alter the consequences of chronic kidney disease after surgery. However, NSS is not suitable for some patients with localized RT due to insufficient parenchyma left, renal vein thrombosis, unfavorable tumor location, and use of anticoagulants (1). Several studies analyzed the national trends for NSS practice over time and the effect of non-tumor-related factors such as the hospital facility or patient characteristics for the choice of NSS in RT $(11,12)$. The trend of increased NSS practice was not universal.

NSS has also been increasingly practiced in Turkey as the treatment of T1 RT in line with recent guidelines. Thus, the aim of this multicentric retrospective study is to investigate the national trends and the effect of non-tumoral factors on the preference of NSS in the treatment of T1 RT in Turkey.

\section{Materials and Methods}

Data was obtained from the Urologic Cancer Database-Kidney Urooncology Association, Turkey (UroCaD-K) which is the largest renal cancer database in Turkey. Study data were collected and managed using REDCap electronic data capture tools hosted at Urooncology Association $(13,14)$ REDCap (Research Electronic Data Capture) is a secure, web-based software platform designed to enable data capture for research studies, providing 1) an intuitive interface for validated data capture; 2) audit trails for tracking data manipulation and export procedures; 3) automated export procedures for seamless data downloads to common statistical packages; and 4) procedures for data integration and interoperability with external sources.

We evaluated the data of 3195 patients who underwent surgical treatment for T1N0M0 RCC at 32 different hospitals in Turkey between 1997-2017. The patients were divided into two groups according to the operation type; RN and NSS groups. The effects of patient-dependent non-tumoral factors such as age, sex, performance score, and hospital-dependent factors such as hospital type, bed size, tumor nephrectomy volume, laparoscopy experience, year of NSS were evaluated. Performance score was defined as ECOG 0 and $\geq 1$. Hospital type was grouped as University Hospital or Non-University Hospital. Hospital volume for $\mathrm{RN}$ or $\mathrm{PN}$ was calculated each year and categorized into four groups across all years $(<50,50-100,100-150, \geq 150$ nephrectomies per year, respectively). In addition, hospital beds were counted and hospitals were categorized into three groups accordingly; small, medium, and large-capacity ( $\leq 500,501$ $999, \geq 1000$ beds, respectively). Patients were grouped in tenyear periods, according to age. Operation dates were grouped in 5-year periods.

\section{Statistical Analysis}

The Stata MP statistical software package (StataCorp, Texas, USA) version 14.2 was used for the analyze collected data. The ShapiroWilk normality test was used to evaluate normal distribution, and a histogram was used to evaluate homogeneity. In the descriptive statistics, mean \pm standard deviation and median (interquartile range) were used. For continuous variables, a t-test was used for data with normal distribution, and the Wilcoxon rank-sum test for data without normal distribution. Fisher's exact test was used to compare categorical variables. The logistic regression method was used for univariate and multivariate analyses. A p-value less than 0.05 was considered to be statistically significant.

This study was approved by Baskent University Institutional Review Board with project number: KA18/221 and supported by Baskent University Research Fund.

\section{Results}

The study cohort included 3.195 patients who underwent surgical intervention at 32 different centers for a renal tumor smaller than $7 \mathrm{~cm}$ diagnosed between 1997 and 2017. Nineteen of the centers were University Hospitals, while thirteen of them were Non-University Hospitals. One of the University Hospitals was small, six of them were medium and twelve of them were large hospitals, while one of the Non-University Hospitals was small, eleven of them were medium and one of them was a large hospital according to their bed counts. Three of the University Hospitals performed $<50,9$ of them 50-100, 6 of them 100150 , one of them $\geq 150$, while 2 of the Non-University Hospitals performed $<50,7$ of them 50-100, 4 of them 100-150 and none of them $\geq 150$ nephrectomies per year.

Among these, 1962 (61.4\%) patients underwent RN, and 1233 (38.6\%) patients underwent NSS, consecutively. The non-tumoral demographic and hospital factors according to procedure types are shown in Table 1. There was a significant increase in the proportion of NSS performed with time, increasing from $9.26 \%$ in the first quarter to $54.78 \%$ in the fourth quarter $(p<0.001)$ (Figure 1). However, there was a significant decrease in NSS practice as the patient age increased $(p<0.001)$. In addition, there was a significant increase in the NSS practice as the hospital size and nephrectomy volume of the hospital increased $(p<0.001)$. However, the practice of NSS was similar according to sex. Univariate and multivariate analyses of nontumoral factors affecting NSS practice between 1997-2017 are presented in Table 2. In general, younger age, recent operation date, larger hospital size with higher nephrectomy volume were parameters that were independently associated to a preference of NSS over RN. Although there was a significant increase in the 
number of NSS performed according to the performance status of the patient and laparoscopic experience of the center in the univariate analysis $(p<0.001)$, no difference was observed in the multivariate analysis of these two parameters.

\section{Discussion}

Our study showed that the NSS ratio for T1 RT increased six-fold in the last two decades in Turkey, similar to other countries in the world. Previously, Hollenbeck et al. (15) had demonstrated an increase in NSS rate from $3.7 \%$ in $1988-1990$ to $12.3 \%$ in 2000 -

\begin{tabular}{|c|c|c|c|}
\hline $\begin{array}{l}\text { Table 1. Non-tumora } \\
\text { according to procedur } \\
\text { Sparing Surgery (RN v }\end{array}$ & $\begin{array}{l}\text { mographic fa } \\
\text { e; Radical Nep } \\
\text { ), between } 199\end{array}$ & $\begin{array}{l}\text { ors of th } \\
\text { rectomy vs } \\
-2018\end{array}$ & $\begin{array}{l}\text { patients } \\
\text { ephron- }\end{array}$ \\
\hline $\begin{array}{l}\text { Procedure type } \\
\% \text { (n) }\end{array}$ & $\begin{array}{l}\text { Radical } \\
\text { Nephrectomy } \\
61.4(1962)\end{array}$ & $\begin{array}{l}\text { Nephron- } \\
\text { Sparing } \\
\text { Surgery } \\
38.6(1233)\end{array}$ & $\mathbf{p}$ \\
\hline Age groups (years)* $\%$ (n) & & & $<0.001$ \\
\hline $41-50$ & $57.11(510)$ & $42.89(383)$ & \\
\hline $51-60$ & $58.23(538)$ & $41.77(386)$ & \\
\hline $61-70$ & $61.22(521)$ & $38.78(330)$ & \\
\hline $71-80$ & $72.81(324)$ & $27.19(121)$ & \\
\hline$>80$ & $84.21(64)$ & $15.79(12)$ & \\
\hline Gendert, \% (n) & & & 0.225 \\
\hline Women & $60.23(748)$ & $39.77(494)$ & \\
\hline Men & $62.37(1205)$ & $37.63(727)$ & \\
\hline Operation date, \% (n) & & & $<0.001$ \\
\hline 1. quarter (1997-2002) & $90.74(294)$ & $9.26(30)$ & \\
\hline 2. quarter (2003-2007) & $76.24(690)$ & $23.76(215)$ & \\
\hline 3. quarter (2008-2012) & $57.22(424)$ & $42.78(317)$ & \\
\hline 4. quarter (2013-2017) & $45.22(554)$ & $54.78(671)$ & \\
\hline Performance status, \% (n) & & & $<0.001$ \\
\hline ECOG 0 & $58.08(1301)$ & $41.92(939)$ & \\
\hline $\mathrm{ECOG}>1$ & $69.21(661)$ & $30.79(294)$ & \\
\hline Hospital type, \% (n) & & & 0.051 \\
\hline Non-University Hospital & $64.25(532)$ & $35.75(296)$ & \\
\hline University Hospital & $60.41(1430)$ & 39.59 (937) & \\
\hline Hospital size, \% (n) & & & $<0.001$ \\
\hline Small $(\leq 500)$ & $74.64(103)$ & $25.36(35)$ & \\
\hline Medium (499-999) & $62.94(866)$ & $37.06(510)$ & \\
\hline Large $(\geq 1000)$ & $59.07(993)$ & $40.9(688)$ & \\
\hline Nephrectomy volume (num & ear), \% (n) & & $<0.001$ \\
\hline$\leq 50$ & $92.16(94)$ & $7.84(8)$ & \\
\hline $50-100$ & $73.13(950)$ & $26.87(349)$ & \\
\hline $100-150$ & $57.61(765)$ & $42.39(563)$ & \\
\hline$\geq 150$ & $32.83(153)$ & $67.17(313)$ & \\
\hline Laparoscopic experience, $\ddagger$ & & & $<0.001$ \\
\hline No & $88.33(159)$ & $11.67(21)$ & \\
\hline Yes & $59.73(1796)$ & $40.27(1211)$ & \\
\hline
\end{tabular}

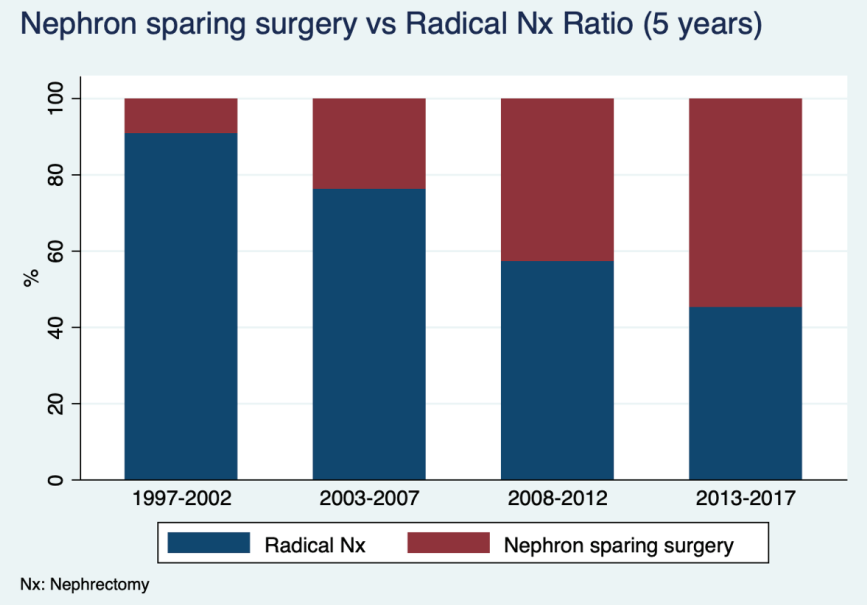

Figure 1. Proportion of NSS practice versus radical nephrectomy over time

2002, regardless of the tumor size. Dulabon et al. (11) analyzed the SEER (Surveillance, Epidemiology and End Results) data in the United States and showed an increase in NSS ratio for T1a RT, from $20 \%$ in 1999 to $45 \%$ in 2006 . Finally, Patel et al. (12) revealed an increase in the frequency of partial nephrectomy nationally from $15.3 \%$ in 2002 to $24.7 \%$ in 2008 (12). Zini et al. (16) on the other hand, revealed an increase from $41 \%$ to $86 \%$ for masses less than $2 \mathrm{~cm}$ and $15 \%$ to $70 \%$ for masses between 2 to $4 \mathrm{~cm}$, from 1987 to 2008 at tertiary European medical centers (16). These increments are certainly influenced by the increased number of incidentally diagnosed small tumors that urologists have been treating over the years. Thus, the surgeons and their centers have been gaining experience and perfecting their skills in NSS. Moreover, the addition of laparoscopic and robotic surgery to the urologic armamentarium has probably caused an increased comprehension of minimally-invasive techniques in urology, resulting in more NSS performed either through the open, laparoscopic, or robot-assisted approach.

We observed a significant decrease in the frequency of NSS as age of patients increased, suggesting that the surgeons preferred RN in older patients. This may be due to an effort to avoid increased time of operation and complications of NSS in older patients with comorbidities. However, such patients are also at an increased risk for baseline renal insufficiency as reported in studies in Europe and United States $(11,12,15,16)$. Although Kim et al. (17) reported a marked increase in the proportion of elderly patients ( $>70$ years) with $\mathrm{T} 1$ renal tumor undergoing NSS from $15.2 \%$ to $27.4 \%$ from $2002-2003$ to $2010-2011$ in the United States and NSS preference was not different with respect to age.

We also noticed alterations in the preference of NSS according to hospital depended on factors such as hospital type, size, and nephrectomy volume. We observed that larger hospitals with higher nephrectomy volume centers like University Hospitals were independently associated NSS practice. Certainly, this is an indicator of the effect of increased experience on the choice of NSS, a more challenging and time-consuming procedure with higher perioperative complication rates than RN (2). Hospital case volume is also known to be a structural indicator of the 
Table 2. The univariate and multivariate analysis of non-tumoral factors affecting the preference of Nephron-Sparing Surgery (NSS) between 1997-2017

\begin{tabular}{|c|c|c|c|c|}
\hline & \multicolumn{2}{|l|}{ Univariate analysis } & \multicolumn{2}{|l|}{ Multivariate analysis } \\
\hline & Odds ratio $(95 \% \mathrm{Cl})$ & $\mathbf{p}$ & Odds ratio $(95 \% \mathrm{Cl})$ & p \\
\hline \multicolumn{5}{|l|}{ Age groups (years) } \\
\hline $51-60$ & $0.95(0.79-1.15)$ & 0.631 & $0.92(0.75-1.13)$ & 0.443 \\
\hline $61-70$ & $0.84(0.69-1.02)$ & 0.081 & $0.75(0.61-0.93)$ & 0.009 \\
\hline \multicolumn{5}{|l|}{ Operation date } \\
\hline 1. quarter (1997-2002) & 1 Reference & & 1 Reference & \\
\hline 2. quarter (2003-2007) & $3.05(2.03-4.58)$ & $<0.001$ & $2.73(1.79-4.18)$ & $<0.001$ \\
\hline 3. quarter (2008-2012) & $7.32(4.90-10.96)$ & $<0.001$ & $5.91(3.77-9.25)$ & $<0.001$ \\
\hline ECOG 0 & $1.62(1.38-1.90)$ & $<0.001$ & $1.15(0.95-1.39)$ & 0.144 \\
\hline \multicolumn{5}{|l|}{ Hospital type } \\
\hline Non-University Hospital & 1 Reference & & 1 Reference & \\
\hline University Hospital & $1.17(0.99-1.38)$ & 0.051 & $1.40(1.10-1.77)$ & 0.005 \\
\hline \multicolumn{5}{|l|}{ Hospital size } \\
\hline Small $(\leq 500)$ & 1 Reference & & 1 Reference & \\
\hline Medium (499-999) & $1.73(1.16-2.58)$ & 0.007 & $1.57(1.32-1.73)$ & $<0.001$ \\
\hline Large $(\geq 1000)$ & $2.04(1.37-3.03)$ & $<0.001$ & $1.55(1.28-1.72)$ & 0.001 \\
\hline \multicolumn{5}{|c|}{ Nephrectomy volume (number/year) } \\
\hline$<50$ & 1 Reference & & 1 Reference & \\
\hline
\end{tabular}

quality of care for many procedures (18). Surgeons working in larger hospitals with higher nephrectomy volumes are more likely to face more-challenging cases and are therefore more prone to attend postgraduate courses and fellowship programs; thereby improve their experience and more-challenging surgical techniques such as like NSS. On the contrary, surgeons working in smaller hospitals with low nephrectomy volumes are unlikely to perform NSS for patients with T1 tumors. Thus, some patients with T1 RT who live in cities away from large-volume hospitals are unlikely to receive the recommended management following urological guidelines. Studies from the United States and Western European countries also revealed similar findings confirming that not every patient was able to receive the optimal treatment for their small RT $(11,12,15)$. Dulabon et al. (11) demonstrated that patients who lived in a rural setting in the United States had significantly lower odds of undergoing NSS than their urban counterparts as tertiary care centers or "centers of excellence" are typically more in metropolitan areas. Also, they concluded that they recognize these disparities to eliminate these biases and ensure the equal delivery of quality healthcare to all patients in the United States. Similar to Dulabonet al. (11), Patel et al. (12) also reported a two-fold difference in NSS rates between urban/teaching hospitals (23.8\%) and rural/nonteaching hospitals (12.3\%), and the large regional differences in the United States (25.4\% in the Northeast vs $18.1-18.5 \%$ elsewhere) demonstrating that high volume nephrectomy centers are more likely to perform NSS for RT than lower volume centers. Moreover, those with private health insurances and higher income were more likely to undergo NSS even after adjusting for age, comorbidity, and a host of hospital factors. According to these results, they concluded that the rising tide has not lifted all boats (12). Hollenbeck et al. (15) revealed that 
patients treated at urban (odds ratio 1.1), teaching (odds ratio 1.3), and high nephrectomy volume (odds ratio 2.5) hospitals were significantly more likely to undergo NSS. Zini et al. (16) presented that institutional NSS is one of the independent factors of the individual probability of treatment of small renal tumor with NSS according to results from six tertiary care centers in Europe.

\section{Study Limitations}

This study has several strengths and limitations. To begin with, this is the first multicentric study investigating the national trends and the effect of non-tumoral factors for the preference of NSS in the treatment of T1 RT in Turkey from 1997 to 2017. However, this is a retrospective study with a smaller sample compared to the literature. The Body Mass Index and prior renal surgery status of the patients were lacking. Also, individual surgeon experience could not be standardized. Although 32 centers were included in our study, this was still a small proportion of the whole health system in Turkey.

\section{Conclusion}

In our multivariate analysis, we observed significant disparity in the use of NSS for T1 RT among the elderly ( $>61$ years), small hospital size ( $\leq 500$ beds), small nephrectomy volume $(<100$ nephrectomies/year), Non-University Hospitals. This disparity could be resolved by persistent education of the residents and urologists with periodic courses and practical training, and by increasing the funds and strengthening the technical equipment of centers. This will lead to NSS been performed even in smaller centers. Thus, this will ensure that suitable patients can be treated with NSS rather than RN in centers other than larger centers.

\section{Acknowledgements}

Publication: This study was presented in the $13^{\text {th }}$ International Urooncology Congress on November 8-12 in 2017, Antalya, Turkey.

Contribution: Güven Aslan, Dokuz Eylül University Faculty of Medicine, Department of Urology; Talha Müezzinoğlu, Celal Bayar University Faculty of Medicine, Department of Urology; Çağ Cal, Ege University Faculty of Medicine, Department of Urology; Levent Türkeri, Acıbadem University Faculty of Medicine, Department of Urology.

Conflict of Interest: No conflict of interest was declared by the authors.

Financial Disclosure: The authors declared that this study received no financial support.

\section{Ethics}

Ethics Committee Approval: This study was approved by Baskent University Institutional Review Board with project number: KA18/221 and supported by Baskent University Research Fund.

Informed Consent: Retrospective study.

Peer-review: Externally peer-reviewed.

\section{Authorship Contributions}

Supervision: S.A., Critical Review: B.A., S.S., Y.B., V.I., E.Ö., O.B., S.B., I.T., Concept: B.K., Ö.Y., T.A.Ö., Design: B.K., Ö.Y., T.A.Ö., Data Collection or Processing: B.K., Ö.Y., T.A.Ö., Analysis or Interpretation: B.K., Ö.Y., T.A.Ö., Literature Search: B.K., Ö.Y., T.A.Ö., Writing: B.K., Ö.Y., T.A.Ö.

\section{References}

1. Ljungberg $B$, Albiges $L$, Bensalah $K$, et al. EAU guidelines on renal cell carcinoma. 2020;1:1-73.

2. Van Poppel H, Da Pozzo L, Albrecht W, et al. A prospective, randomised EORTC intergroup phase 3 study comparing the oncologic outcome of elective nephron-sparing surgery and radical nephrectomy for low-stage renal cell carcinoma. Eur Urol 2011;59:543-552.

3. Arnold ML, Thiel DD, Diehl N, et al. Comparison of baseline quality of life measures between renal cell carcinoma patients undergoing partial versus radical nephrectomy. BMC Urol 2013;13:52.

4. Pierorazio PM, Johnson $\mathrm{MH}$, Patel HD, et al. Management of renal masses and localized renal cancer: systematic review and metaanalysis. J Urol 2016;196:989-999.

5. Huang WC, Elkin EB, Levey AS, et al. Partial nephrectomy versus radical nephrectomy in patients with small renal tumors--is there a difference in mortality and cardiovascular outcomes? I Urol 2009;181:55-61; discussion 61-52.

6. Kates $M$, Badalato GM, Pitman M, et al. Increased risk of overall and cardiovascular mortality after radical nephrectomy for renal cell carcinoma $2 \mathrm{~cm}$ or less. J Urol 2011;186:1247-1253.

7. Weight C], Larson BT, Fergany AF, et al. Nephrectomy induced chronic renal insufficiency is associated with increased risk of cardiovascular death and death from any cause in patients with localized $\mathrm{cT1} 1 \mathrm{~b}$ renal masses. J Urol 2010;183:1317-1323.

8. Scosyrev E, Messing EM, Sylvester R, et al. Renal function after nephron-sparing surgery versus radical nephrectomy: results from EORTC randomized trial 30904. Eur Urol 2014;65:372-377.

9. Poulakis V, Witzsch $U$, de Vries $R$, et al. Quality of life after surgery for localized renal cell carcinoma: comparison between radical nephrectomy and nephron-sparing surgery. Urology 2003;62:814-820.

10. Huang WC, Donin NM, Levey AS, et al. Chronic kidney disease and kidney cancer surgery: new perspectives. J Urol 2020;203:475-485.

11. Dulabon LM, Lowrance WT, Russo $P$, et al. Trends in renal tumor surgery delivery within the United States. Cancer 2010;116:2316-2321.

12. Patel SG, Penson DF, Pabla B, et al. National trends in the use of partial nephrectomy: a rising tide that has not lifted all boats. J Urol 2012;187:816-821.

13. Harris PA, Taylor R, Thielke R, et al. Research electronic data capture (REDCap)--a metadata-driven methodology and workflow process for providing translational research informatics support. J Biomed Inform 2009; 42:377-381.

14. Harris PA, Taylor R, Minor BL, et al. The REDCap consortium: building an international community of software platform partners. J Biomed Inform 2019;95:103208.

15. Hollenbeck BK, Taub DA, Miller DC, et al. National utilization trends of partial nephrectomy for renal cell carcinoma: a case of underutilization? Urology 2006;67:254-259.

16. Zini L, Patard JJ, Capitanio U, et al. The use of partial nephrectomy in European tertiary care centers. Eur J Surg Oncol 2009;35:636-642.

17. Kim SP, Gross CP, Meropol N, et al. National treatment trends among older patients with T1-localized renal cell carcinoma. Urol Oncol 2017;35:113.e15-113.e21. doi: 10.1016/j.urolonc.2016.10.008.

18. Birkmeyer JD, Stukel TA, Siewers AE, et al. Surgeon volume and operative mortality in the United States. N Engl J Med 2003;349:2117-2127. 